\title{
CORRELATION PROPERTIES OF INTERFERING ELECTRONS IN A MESOSCOPIC RING UNDER NONCLASSICAL MICROWAVE RADIATION
}

\author{
D. I. TSOMOKOS, C. C. CHONG AND A. VOURDAS \\ Department of Computing, School of Informatics, \\ University of Bradford, \\ Bradford BD7 1DP, United Kingdom
}

\begin{abstract}
Interfering electrons in a mesoscopic ring are irradiated with both classical and nonclassical microwaves. The average intensity of the charges is calculated as a function of time and it is found that it depends on the nature of the irradiating electromagnetic field. For various quantum states of the microwaves, the electron autocorrelation function is calculated and it shows that the quantum noise of the external field affects the interference of the charges. Two-mode entangled microwaves are also considered and the results for electron average intensity and autocorrelation are compared with those of the corresponding separable state. In both cases, the results depend on whether the ratio of the two frequencies is rational or irrational.
\end{abstract}

\section{Introduction}

The Aharonov-Bohm effect [1] manifests itself as a nontrivial quantum phase, whenever electric charges travel in a field-free region enclosing a magnetostatic flux. This 'geometrical phase' has been generalized [2] and the original results have found applications in various contexts, for example in conductance oscillations in mesoscopic rings [3] and 'which-path' experiments that use novel solid-state devices [4].

A recent development of these ideas has been to replace the magnetostatic flux by an electromagnetic field [5]. The objective here is very different, since this 'ac Aharonov-Bohm experiment' constitutes a nonlinear device where the interaction between the interfering electrons and the photons leads to interesting nonlinear phenomena [6]. For an overview of related studies on the interaction of mesoscopic devices with microwaves we refer the reader to [7].

It is interesting to investigate the same phenomena with quantized electromagnetic fields. This 'quantum ac Aharonov-Bohm experiment' with nonclassical microwaves, has been studied [5] and one can quantify how the 
quantum noise destroys slightly the electron interference [8]. The aim is to investigate how various quantum phenomena and the quantum statistics of the nonclassical microwaves link to corresponding quantum phenomena on the electrons.

In what follows we study the interference of the electrons by calculating their intensity, while they are being irradiated with classical or nonclassical microwaves. The correlation properties of electron interference are then studied by calculating the autocorrelation function of the electron intensity (Sec. 2). We also consider two-mode microwaves with frequencies $\omega_{1}$ and $\omega_{2}$ (Sec. 3). Two-mode microwaves can be factorizable, separable or entangled [9] and since the problem of entanglement is generally complex, we approached it using an example. In particular, we assumed that the two modes of the microwave field form a Bell state and calculated its effect on electron interference. We found that the result is very different from that of the corresponding separable case. We conclude in Sec. 4 with a discussion of our results.

\section{One-mode microwaves}

\subsection{Classical microwaves}

The following system is considered: a beam of electric charges splits into two possible paths $C_{0}$ and $C_{1}$. The charges enter a region that is irradiated with microwaves (using a suitable waveguide). The microwaves propagate in the waveguide with the time-dependent magnetic field perpendicular to the plane of the two paths and the electric field parallel to it. Let $\psi_{0}, \psi_{1}$ be the electron wavefunctions with total winding equal to 1 , in the absence of magnetic field. The effect of the electromagnetic field is the phase factor $\exp [i e \phi(t)]$ and the intensity is

$I(t)=\left|\psi_{0}+\psi_{1} \exp [i e \phi(t)]\right|^{2}=\left|\psi_{0}\right|^{2}+\left|\psi_{1}\right|^{2}+2\left|\psi_{0}\right|\left|\psi_{1}\right| \Re\{\exp [i(\sigma+e \phi(t))]\}(1)$

where $\sigma=\arg \left(\psi_{1}\right)-\arg \left(\psi_{0}\right)$. Units in which $k_{B}=\hbar=c=1$ are used throughout. For simplicity we consider the case of equal splitting, in which $\left|\psi_{0}\right|^{2}=\left|\psi_{1}\right|^{2}=1 / 2$ and let $\sigma=0$. In this case we get

$$
I(t)=1+\cos [e \phi(t)]
$$

We calculate the autocorrelation function of the electron intensity:

$$
\Gamma(\tau)=\lim _{T \rightarrow \infty} \frac{1}{2 T} \int_{-T}^{T} R(t, \tau) d t ; \quad R(t, \tau) \equiv I(t) I(t+\tau) .
$$


An expansion of $\Gamma(\tau)$ into a Fourier series gives the spectral density $S_{K}$ :

$$
\begin{aligned}
S_{K} & =\frac{\Omega}{2 \pi} \int_{0}^{2 \pi / \Omega} \Gamma(\tau) \exp (-i K \Omega \tau) d \tau \\
\Gamma(\tau) & =\sum_{K=-\infty}^{\infty} S_{K} \exp (i K \Omega \tau) .
\end{aligned}
$$

Firstly, we consider the case where the classical time-dependent flux is given by

$$
\phi(t)=\phi_{1} \sin (\omega t)
$$

and using Eqs. (2) and (3) we find the autocorrelation function:

$$
\Gamma_{c l}(\tau)=\left[1+J_{0}\left(e \phi_{1}\right)\right]^{2}+2 \sum_{K=1}^{\infty}\left[J_{2 K}\left(e \phi_{1}\right)\right]^{2} \cos (2 K \omega \tau),
$$

where $J_{K}$ are Bessel functions. Comparison of Eqs. (4) and (6) shows that $\Omega=2 \omega$ and

$$
S_{0}=\left[1+J_{0}\left(e \phi_{1}\right)\right]^{2} ; \quad S_{K}=\left[J_{2 K}\left(e \phi_{1}\right)\right]^{2} .
$$

\subsection{Nonclassical microwaves}

A monochromatic electromagnetic field of frequency $\omega$ is considered, at temperatures $k_{B} T<<\hbar \omega$. We quantize the electromagnetic field by considering the vector potential $A_{i}$ and the electric field $E_{i}$ as dual quantum variables. The loop $C=C_{0}-C_{1}$ is small in comparison to the wavelength of the microwaves, hence the $A_{i}$ and the $E_{i}$ can be integrated around it and yield the magnetic flux $\phi$ and the electromotive force $V_{\mathrm{EMF}}$, respectively, as dual quantum variables. The annihilation operator can be introduced as $a=2^{-\frac{1}{2}} \xi^{-1}\left(\phi+i \omega^{-1} V_{\mathrm{EMF}}\right)$, and similarly the creation operator, where $\xi$ is a constant proportional to the area enclosed by $C$. The flux operator is consequently written as $\phi(t)=\exp (i t H) \phi(0) \exp (-i t H)$, where $H$ is the Hamiltonian that contains the $\omega a^{\dagger} a$ term and an interaction term. This interaction term can be neglected for small currents.

Under these conditions the magnetic flux, which defines the phase factor, becomes the operator $\hat{\phi}(t)=(\xi / \sqrt{2})\left[\exp (i \omega t) a^{\dagger}+\exp (-i \omega t) a\right]$. Hence this phase factor $\exp (i e \phi)$ now is

$$
\exp [i e \hat{\phi}(t)]=D[i q \exp (i \omega t)], \quad q=\frac{\xi e}{\sqrt{2}}
$$

where $D(\lambda)$ is the displacement operator $D(\lambda)=\exp \left(\lambda a^{\dagger}-\lambda^{*} a\right)$. The interference between the two electron beams is described by the intensity 
operator

$$
\hat{I}(t)=1+\cos [e \hat{\phi}(t)]=1+\frac{1}{2} D[i q \exp (i \omega t)]+\frac{1}{2} D[-i q \exp (i \omega t)] .
$$

Let $\rho$ be the density matrix describing the external nonclassical microwaves. The expectation value of the electron intensity is

$$
\langle I(t)\rangle \equiv \operatorname{Tr}[\rho \hat{I}(t)]=1+\frac{1}{2} \tilde{W}(\lambda)+\frac{1}{2} \tilde{W}(-\lambda) ; \quad \lambda=i q \exp (i \omega t),
$$

where $\operatorname{Tr}[\rho D(\lambda)] \equiv \tilde{W}(\lambda)$ is the Weyl (or characteristic) function which has been studied by various authors including ourselves (e.g. [10] and references therein).

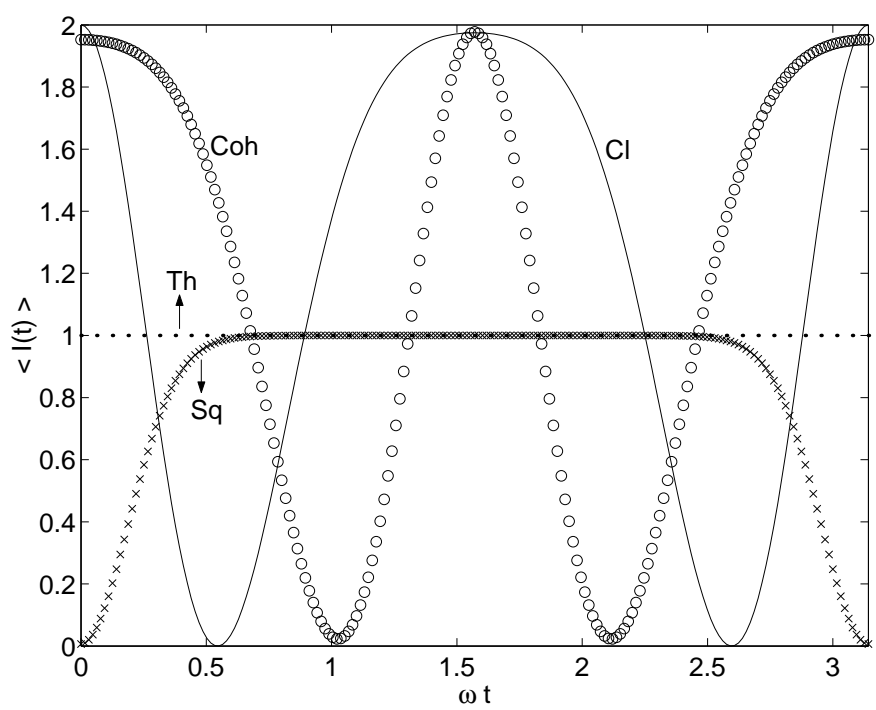

Figure 1. $\langle I(t)\rangle$ as a function of $\omega t$ for $\omega=10^{-4},\langle N\rangle=200, r=6.4$. We use units where $\hbar=k_{B}=c=1$. Continuous line represents the case of irradiation with classical microwaves; line of circles, coherent states; line of crosses, squeezed states; and dotted line, thermal states.

We have calculated $\langle I(t)\rangle$ for various quantum states of the microwaves (using results for $\tilde{W}(\lambda)$ in Ref. [11]). In order to find the $\Gamma(\tau)$ from Eq. (3), one needs to calculate the quantity

$$
R(t, \tau) \equiv \operatorname{Tr}\left[\rho \hat{I}^{\dagger}(t) \hat{I}(t+\tau)\right]
$$

Numerical results are presented for different quantum states that we calculated. In particular, we plot four cases: classical microwaves and 

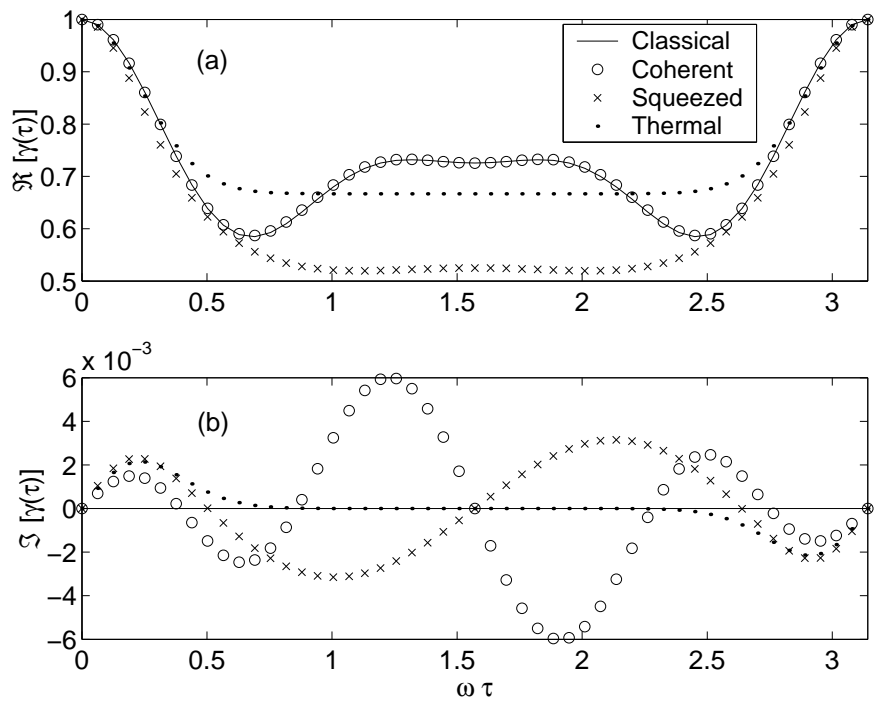

Figure 2. $\gamma(\tau)$ as a function of $\omega \tau$ for $\omega=10^{-4},\langle N\rangle=200, r=6.4$. Part (a) shows the real part of $\gamma(\tau)$; part (b) shows the imaginary part. We use units where $\hbar=k_{B}=c=1$.

nonclassical microwaves in coherent, squeezed, and thermal states. For a meaningful comparison, we consider the case where the average number of photons $\langle N\rangle$ in coherent, squeezed, and thermal states is the same:

$$
\begin{aligned}
\langle N\rangle=|A|^{2} & =\left[\sinh \left(\frac{r}{2}\right)\right]^{2}+\left[\cosh \left(\frac{r}{2}\right)-\sinh \left(\frac{r}{2}\right)\right]^{2} B^{2} \\
& =\frac{1}{\exp (\beta \omega)-1}
\end{aligned}
$$

For the classical case we took $\phi_{1}^{2}=2|A|^{2}=2\langle N\rangle$. In all results of Figs. 1 to $3, \omega=10^{-4}$ (which in our units is $e V$ ), $\langle N\rangle=200, r=6.4$.

The results show that the quantum noise in the irradiating microwaves affects the electron interference. All microwaves that we have considered have the same average number of photons, but differ in the quantum noise. These four types of microwaves lead to different electron interference results and different autocorrelation functions. Irradiation of the electrons by nonclassical microwaves leads to nonzero value of the imaginary part of the electron autocorrelation function. This is not so (i.e. the imaginary part of $\Gamma(\tau)$ vanishes) when the ring is irradiated with classical microwaves. 


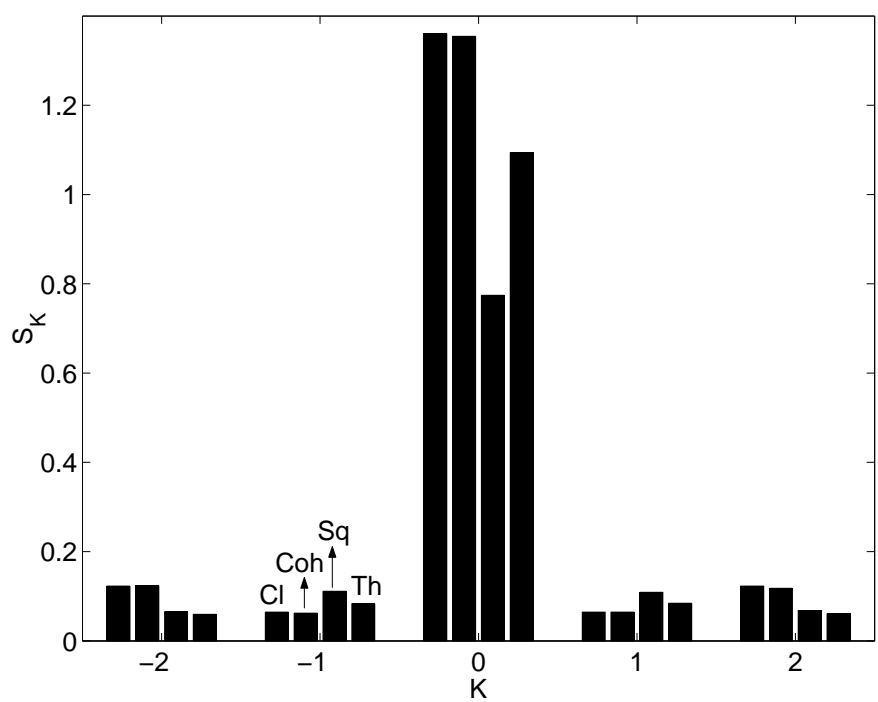

Figure 3. $S_{K}$ coefficients for the electrons with $\langle N\rangle=200, r=6.4$. We use units where $\hbar=k_{B}=c=1$. The bars correspond to irradiation of the ring by (from left to right): classical, coherent, squeezed, and thermal states.

\section{Two-mode nonclassical microwaves}

We consider two-mode nonclassical microwaves. We are particularly interested to study how entangled two-mode microwaves affect the electron interference. For this reason we consider a Bell state $|s\rangle=2^{-1 / 2}(|01\rangle+|10\rangle)$ where $|01\rangle,|10\rangle$ are two mode number eigenstates. For comparison we also consider the separable (disentangled) state

$$
\rho_{\text {sep }}=\frac{1}{2}(|01\rangle\langle 01|+| 10\rangle\langle 10|) .
$$

Clearly, the density matrix of the entangled state $\rho_{\text {ent }}=|s\rangle\langle s|$ can be written as

$$
\rho_{\text {ent }}=\rho_{\text {sep }}+\frac{1}{2}(|01\rangle\langle 10|+| 10\rangle\langle 01|) .
$$

In this case the phase factor $\exp [i e \phi(t)]$ becomes the product of two displacement operators and, consequently, the intensity becomes

$$
\hat{I}(t)=1+\frac{1}{2} D_{1}\left(\lambda_{1}\right) D_{2}\left(\lambda_{2}\right)+\frac{1}{2} D_{1}\left(-\lambda_{1}\right) D_{2}\left(-\lambda_{2}\right) ; \lambda_{j}=i q \exp \left(i \omega_{j} t\right)
$$

for two modes $(j=1,2)$. Therefore, we find that

$$
\begin{aligned}
& \langle I(t)\rangle_{\text {sep }}=1+\left(1-q^{2}\right) \exp \left(-q^{2}\right), \\
& \langle I(t)\rangle_{\text {ent }}=\langle I(t)\rangle_{\text {sep }}-q^{2} \exp \left(-q^{2}\right) \cos \left[\left(\omega_{1}-\omega_{2}\right) t\right] .
\end{aligned}
$$


It is seen that for this example, the $\langle I(t)\rangle_{\text {sep }}$ is constant in time, while the $\langle I(t)\rangle_{\text {ent }}$ is an oscillatory function of time. Clearly, different correlations among the two irradiating modes of the microwaves may lead to different average electron intensities.

\section{Discussion}

The subject of mesoscopic devices interacting with microwaves has received attention in the last few years (e.g., Ref. [7]). Our contribution has been to consider that these microwaves are prepared in various nonclassical states $[5,8,12]$. Here we have quantified the effect of the quantum noise on electron interference. More specifically we have calculated both the electron average intensity and the spectral density for several types of nonclassical microwaves and a comparison of the results with the case of classical microwaves (Figs. 1-3), demonstrates clearly that the presence of both classical and quantum noise in the nonclassical microwaves affects the electron intensity. What is more, when the ring is irradiated with two-mode microwaves, then entanglement among these two modes (i.e., the formation of a Bell state) leads to a time-dependent expectation value of the electron intensity.

\section{References}

1. M. Peshkin and A. Tonomura, The Aharonov-Bohm effect, Lecture notes in Physics Vol. 340, Berlin: Springer (1989).

2. A. Shapere and F. Wilczek (ed), Geometric Phases in Physics, Singapore: World Scientific (1989).

3. S. Washburn and R.A. Webb, Adv. Phys. 35, 375 (1986)

A.G. Aronov and Y.V. Sharvin, Rev. Mod. Phys. 59, 755 (1987).

4. G. Hackenbroich, Phys. Rep. 343, 464 (2001).

5. A. Vourdas, Phys. Rev. B54, 13175 (1996)

A. Vourdas and B.C. Sanders, Europhys. Lett. 43, 659 (1998).

6. M.P. Silverman, Nuovo Cimento B97, 200 (1987)

M. Buttiker, Phys. Rev. B46, 12485 (1992).

7. M. Buttiker, J. Low Temp. Phys. 118, 519 (2000)

R. Deblock et. al., Phys. Rev. B65, 075301 (2002).

8. P. Cedraschi, V.V. Ponomarenko, M. Buttiker, Phys. Rev. Lett. 84, 346 (2000) A. Vourdas, Phys. Rev. A64, 053814 (2001).

9. R.F. Werner, Phys. Rev. A40, 4277 (1989); A. Peres, Phys. Rev. Lett. 77, 1413 (1996); R. Horodecki and M. Horodecki, Phys. Rev. A54, 1838 (1996); V. Vedral et. al., Phys. Rev. Lett. 78, 2275 (1997).

10. S. Chountasis and A. Vourdas, Phys. Rev. A58, 848 (1998).

11. A. Vourdas, Phys. Rev. B49, 12040 (1994).

12. C.C. Chong, D.I. Tsomokos, A. Vourdas, Phys. Rev. A66, 33813 (2002). 